\title{
Aplicación de la escarificación física y mecánica en la emergencia y crecimiento de semillas de tara (Caesalpinia spinosa)
}

\section{Application of physical and mechanical scarification in the emergence and growth of tara seeds (Caesalpinia spinosa)}

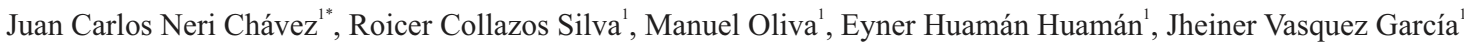

\section{RESUMEN}

La presente investigación se llevó a cabo en el área del vivero de la UNTRM, distrito de Chachapoyas, cuyo objetivo fue evaluar los métodos de escarificación física y mecánica en la emergencia y crecimiento de semillas de Caesalpinia spinosa. Se utilizó un diseño completo al azar (DCA), con tres repeticiones y ocho tratamientos. Para el análisis de datos se utilizó el software R x 643.3 .1 y la prueba de Duncan al 5\%. Los tratamientos fueron: remojada en agua fría por 3 días (T1), remojada en agua fría por 5 días (T2), remojada en agua fría por 7 días (T3), picada y remojada por 12 horas (T4), picada y remojada por 24 horas (T5), remojada en agua caliente por 1 minuto y en agua fría por 12 horas (T6), remojada en agua caliente por 3 minutos y en agua fría por 12 horas (T7) y remojada en agua caliente por 5 minutos y en agua fría por 12 horas (T8). Las semillas se sembraron en sustrato a base de arena de río. Se aplicó riegos de 2-3 veces por semana. Las variables evaluadas fueron porcentaje de emergencia, altura de plántula, número de hojas, longitud de raíz e índice de velocidad de emergencia. Según los resultados obtenidos existieron diferencias significativas entre tratamientos donde el T4 y T5 lograron mayores porcentajes de emergencia con 91.67 y $93.75 \%$, altura de plántula con 7.85 y $8.97 \mathrm{~cm}$ y número de hojas con 3.70 y 3.87 . Igualmente para el índice de velocidad de emergencia los valores más altos se obtuvieron en el T4 y T5 con 14.16 y 14.85 respectivamente, demostrándose así el efecto notorio de la escarificación mecánica sobre la emergencia y características morfológicas de plántulas de tara.

Palabras claves: Escarificación, semilla, emergencia, Caesalpinia spinosa.

\begin{abstract}
The present investigation was carried out in the nursery area of the UNTRM, district of Chachapoyas, whose objective was to evaluate the methods of physical and mechanical scarification in the emergence and growth of seeds of Caesalpinia spinosa. A complete randomized design (DCA) was used, with three repetitions and eight treatments. The software R x 643.3 .1 and the Duncan test at 5\% were used for the data analysis. The treatments were: soaked in cold water for 3 days (T1), soaked in cold water for 5 days (T2), soaked in cold water for 7 days (T3), minced and soaked for 12 hours (T4), chopped and soaked for 24 hours (T5), soaked in hot water for 1 minute and in cold water for 12 hours (T6), soaked in hot water for 3 minutes and in cold water for 12 hours (T7) and soaked in hot water for 5 minutes and in cold water for 12 hours (T8). The seeds were sown on a substrate based on river sand. Irrigations were applied 2-3 times per week. The evaluated variables were emergency percentage, seedling height, number of leaves, root length and emergency speed index. According to the results obtained there were significant differences between treatments where T4 and T5 achieved higher emergency percentages with 91.67 and $93.75 \%$, seedling height with 7.85 and $8.97 \mathrm{~cm}$ and number of leaves with 3.70 and 3.87. Igualmente para el índice de velocidad de emergencia los valores más altos se obtuvieron en el T4 y T5 con 14.16 y 14.85 respectivamente, demostrándose así el efecto notorio de la escarificación mecánica sobre la emergencia y características morfológicas de plántulas de tara.
\end{abstract}

Keywords: Scarification, seed, emergence, Caesalpinia spinosa.

\footnotetext{
${ }^{1}$ Universidad Nacional Toribio Rodríguez de Mendoza de Amazonas (UNTRM-A), Instituto de Investigación para el Desarrollo Sustentable de Ceja de Selva, Calle Higos Urco N³42-350-356, Calle Universitaria N³04, Chachapoyas, Perú

*Autor de correspondencia. E-mail: jneri@indes-ces.edu.pe
} 


\section{INTRODUCCIÓN}

Dentro de la familia Fabaceae, el género Caesalpinia, abarca 150 especies, de las que 40 están presentes en Sudamérica. Caesalpinia spinosa, es un árbol verde, con espinas en tallo, ramas y hojas, alcanzando de los 3 a los 8 metros de altura y sus flores se disponen en racimos terminales. El fruto, es una legumbre con la testa dura, de 6 a $10 \mathrm{~cm}$ de largo y con 5 a 8 semillas redondas. Es conocido con los nombres de "tara", "guaranga", "tanino" o "taya" (Dostert et al., 2009; Cordero et al., 2017).

En Perú, se encuentra la mayor área de bosques de tara, seguido por Bolivia, Chile, Ecuador y Colombia (Castell-Escuer, 2012), y es considerado el principal productor mundial de tara con más del $80 \%$ de la producción mundial. Sin embargo no logra cubrir la demanda internacional por lo que varios países se han interesado en su comercio (Dodds, 2015). Esto se debe a que la mayor producción proviene de plantas silvestres, es decir, la producción en el Perú es básicamente de bosques naturales y en algunas zonas en parcelas agroforestales. El principal uso de las legumbres y semillas de la tara, es con como fuente de gomas y taninos ( $40 \mathrm{a}$ $60 \%$ ) hidrolizables con ácido gálico como el principal constituyente (Aguilar-Galvez et al., 2014).

La germinación de semillas se efectúa en tres etapas, incrementando su metabolismo celular y restaurando el crecimiento del embrión, luego se da la rotura de la cubierta seminal y la salida de la plántula (Bewley, 1997). Primero se produce la imbibición que facilita la entrada del agua hacia la semilla, luego se activan enzimas y finalmente se produce la síntesis de proteínas, permitiendo la formación de sustancias necesarias para el desarrollo de la semilla (Trigiano y Gray, 2011).

Cuando no germinan las semillas es debido a dos motivos, uno la quiescencia, que se produce cuando el medio no es favorable para el crecimiento vegetativo debido a la baja humedad o temperaturas no adecuadas. Y el otro, la latencia, que viene a ser un bloqueo del proceso de la germinación de semillas intactas y viables (Baskin y Baskin, 2004), aunque se les brinden las condiciones de temperatura, luz, humedad y concentración adecuada de oxígeno para germinar (Doria, 2010). Existen varios tipos de latencia, que se determinan según la geminación delas semillas. Una de ellas es la latencia exógena o de cubierta, dándose cuando la testa es muy dura o impermeable (Finch-Savage y Leubner-Metzger, 2006). Otra es la latencia morfológica que se produce cuando lo embriones son inmaduros y requieren tiempo para la germinación (Baskin y Baskin, 2013).

Se han desarrollado varios métodos para romper la latencia y estimular la germinación como es la escarificación física (aplicando calor seco o húmedo), mecánica y química (Baskin y Baskin, 2013). En la escarificación física, existen tratamientos pregermi-nativos mediante el proceso de imbibición, que se aplican con la finalidad de homogenizar la germi-nación de las semillas, sobretodo en especies cuyas semillas tardan en germinar (Coronel de Renolfi et al., 2013; Oliva et al., 2014). Otro tratamiento es el ablandamiento de la testa de la semilla por medio de la inmersión en agua caliente, esto influye en la ruptura de latencia física de las semillas de las Fabaceae (Sánchez-Paz y RamírezVillalobos, 2006). La escari-ficación mecánica, en cambio consiste en friccionar cualquier material áspero sobre la semilla, como el uso de papel lija y arena gruesa (Atencio et al., 2003). En semillas de Caesalpinia spinosa, (Rossini-Oliva et al., 2006) lograron un $100 \%$ de germinación de semillas, mientras que (Ortega-Baes et al., 2001) obtuvieron un 44,67\% de semillas germinadas en Caesalpinia paraguariensis. Por otra parte, cortando la testa de semillas de Caesalpinia cacalaco (Alvarez-Aquino et al., 2014) tuvieron un $98,5 \%$ de germinación.

Dada la importancia que constituye la especie C. espinosa en la parte productiva y forestal, en este trabajo se busca contribuir con el desarrollo de una metodología para su propagación, incrementando el porcentaje y la velocidad de emergencia de las plántulas mediante la aplicación de escarificación física con inmersiones en agua fría y/o caliente, y escarificación mecánica mediante cortes en la testa de las semillas. 


\section{MATERIALY MÉTODOS}

\section{Ubicación del experimento}

La investigación, se ejecutó entre los meses de setiembre a noviembre en el vivero de la Estación Experimental Chachapoyas, dentro del campus universitario de la Universidad Nacional Toribio Rodríguez de Mendoza (UNTRM), a una altitud de 2334 m.s.n.m., con una temperatura promedio es de $18^{\circ} \mathrm{C}$ y una humedad relativa de $74 \%$. La zona presenta un clima variado y templado, las lluvias se presentan mayormente en los meses de diciembre a abril con promedio de $811 \mathrm{~mm}$.

\section{Preparación del sustrato}

Se utilizó un sustrato a base de arena cernida de río, previamente desinfectado con formol (1 litro en 20 litros de agua) y luego se cubrió con una bolsa plástica durante 4 días.

\section{Acondicionamiento del área experimental}

Se construyó una cama almaciguera cubierta con malla Rachel, ubicada a 1,50 m sobre la superficie del suelo con dimensiones de $1 \mathrm{~m}$, de ancho, 2,16 m de largo y $0,30 \mathrm{~m}$ de profundidad.

Luego se procedió a dividir y trazar las áreas para las 24 unidades experimentales con ayuda de un cordel.

\section{Diseño del experimento}

En este experimento se usó un Diseño en Completo al Azar (DCA), en un área total de 2,16 m², con 1920 semillas de tara sembradas, 3 repeticiones y 8 tratamientos. Cada unidad experimental tuvo un área de $0.089 \mathrm{~m}^{2}(0,27 \times 0,33 \mathrm{~m})$ con 8 surcos y cada surco con 10 semillas sembradas. Posteriormente, para las evaluaciones se consideró un total de 10 plántulas emergidas de la parte central para evitar efectos del borde.

\section{Tratamientos}

De acuerdo a los tratamientos donde se involucró a la escarificación física y mecánica se emplearon los tratamientos que observamos en la Tabla 1 .
Tabla 1. Tratamientos de escarificación física (EF) y mecánica (EM) en semillas de tara

\begin{tabular}{|c|c|c|c|}
\hline $\mathbf{T}$ & Descripción & Tipo & Tiempo \\
\hline 1 & Remojada en agua fría & $\mathrm{EF}$ & 3 días \\
\hline 2 & Remojada en agua fría & EF & 5 días \\
\hline 3 & Remojada en agua fría & $\mathrm{EF}$ & 7 días \\
\hline 4 & Picada y remojada & $\mathrm{EM}$ & 12 horas \\
\hline 5 & Picada y remojada & EM & 24 horas \\
\hline 6 & $\begin{array}{c}\text { Remojada en agua caliente a } \\
\mathrm{T}^{\circ} \text { de ebullición }\end{array}$ & $\mathrm{EF}$ & $\begin{array}{l}1 \text { minuto y remojada } \\
\text { en agua fría/ } 12 \text { horas }\end{array}$ \\
\hline 7 & $\begin{array}{c}\text { Remojada en agua caliente a } \\
\mathrm{T}^{\circ} \text { de ebullición }\end{array}$ & $\mathrm{EF}$ & $\begin{array}{l}3 \text { minutos y remojada } \\
\text { en agua fría } / 12 \text { horas }\end{array}$ \\
\hline 8 & $\begin{array}{c}\text { Remojada en agua caliente a } \\
\mathrm{T}^{\circ} \text { de ebullición }\end{array}$ & $\mathrm{EF}$ & $\begin{array}{l}5 \text { minutos yremojada } \\
\text { en agua fría } / 12 \text { horas }\end{array}$ \\
\hline
\end{tabular}

\section{Recolección de las semillas}

Las semillas de tara, fueron recolectadas en el distrito de la Jalca, al menos 10 a 20 plantas jóvenes, vigorosas $\mathrm{y}$ en buen estado fitosanitario. El número de vainas recolectadas por árbol fue la misma cantidad.

Obtención de las semillas y aplicación de los tratamientos

Tras la recolección, se procedió a desvainar para obtener las semillas de tara, seleccionando las de un tamaño uniforme evitando aquellas de las partes extremas. Tras tener un promedio de 2000 semillas se procedió a la aplicación de los tratamientos de escarificación física (remojo en agua fría y hervida) y mecánica (corte de la testa) de acuerdo al Tabla 1 .

\section{Siembra de las semillas}

En la almaciguera con el sustrato preparado, se sembraron de forma directa para todo el experimento un total 1920 semillas tratadas (80 por tratamiento y 640 por repetición). Para la siembra de las semillas se consideró una profundidad de 2 veces su espesor y se cubrió con una capa de arena.

Riego

Después de la siembra se aplicó un riego ligero, para dar las condiciones necesarias para el inicio de la germinación y posterior emergencia de las plántulas. Posteriormente el riego se aplicó de 2 a 3 veces por semana de acuerdo a las condiciones climáticas de la zona y necesidad de las plántulas.

\section{Medición de las variables}

Porcentaje de emergencia de plántulas (PE)

Para el porcentaje de emergencia de las plántulas de tara se realizó un seguimiento desde la siembra y se evaluó hasta que emerjan más del $50 \%$ de las plántulas. 
Se determinó con la siguiente fórmula (Cárdenas 2011):

$$
P E=\frac{\text { semillas germinadas }}{\text { semillas totales }} * 100
$$

\section{Índice de velocidad de emergencia (IVE)}

Se midió el índice de la tasa de emergencia (Cárdenas 2011), es decir la velocidad de emergencia de acuerdo al número total de semillas germinadas por un período de tiempo donde la mayor parte de las plántulas emergieron (dos meses), mediante la siguiente fórmula:

$$
I^{\prime} V E=N P / D+N P / D+\ldots+N P / D
$$

Donde:

$N P=$ número de plántulas emergidas

$D=$ días después de la siembra

\section{Altura de plántula $(\mathrm{cm})$}

La evaluación de altura de plántula se realizó a 10 plántulas por tratamiento, desde la base del tallo hasta elápice de cada una de ellas.

\section{Número de hojas}

Para el número de hojas se evaluó 10 plántulas contando las hojas compuestas en cada una. Se consideró solo las hojas verdaderas.

\section{Longitud de raíz (cm)}

La longitud de raíz igualmente se realizó la evaluación en 10 plántulas midiendo la raíz de cada planta.

Cabe mencionar que las evaluaciones de las variables se llevaron a cabo hasta que las plántulas registraron de 6-9 cm, cuando están ya listas para el trasplante a campo, o sea unos 2 meses

\section{Procesamiento de datos}

El procesamiento de los datos para las variables porcentaje de germinación, índice de velocidad de emergencia, altura y longitud de plántula se utilizó el software R x 64 3.3.1 (referencia, poner la de otro nuemro) aplicando la prueba de Duncan al 5\% de significación.

\section{RESULTADOS}

\section{Porcentaje de emergencia de plántulas}

En la variable porcentaje de germinación de plántulas según el análisis de varianza con la prueba de Duncan $(p<0,05)$ existieron diferencias altamente significativas entre los tratamientos de escarificación cuyos datos de porcentaje fueron transformados a la función $\sqrt{ } \mathrm{X}+5$ para garantizar que pertenezcan a una distribución normal, además presentaron un coeficiente de variación de $28,97 \%$ lo que indica la confiabilidad de los datos en experimentos agrícolas (Patel et al., 2001) (Tabla 2).

Tabla 2. Prueba de medias para la variable porcentaje de emergencia de plántulas $10 \mathrm{~cm}$

\begin{tabular}{cccc}
\hline Tratamiento & Media & Media $\sqrt{ } \mathrm{X}+5$ & Rango \\
\hline T5 & 93.75 & 4.45 & $\mathrm{~A}$ \\
T4 & 91.67 & 4.38 & $\mathrm{~A}$ \\
T3 & 28.75 & 2.65 & $\mathrm{~B}$ \\
T2 & 23.75 & 2.62 & $\mathrm{~B}$ \\
T1 & 20.42 & 2.55 & $\mathrm{~B}$ \\
T6 & 20.42 & 2.50 & $\mathrm{BC}$ \\
T7 & 9.58 & 2.36 & $\mathrm{BC}$ \\
T8 & 4.17 & 2.28 & $\mathrm{D}$ \\
\hline
\end{tabular}

Según los resultados obtenidos en la Figura 1 en la escarificación mecánica en los tratamientos 4 y 5 donde la testa fue cortada, se lograron los mayores valores promedios de porcentaje de emergencia de plántulas con 4,38 (91,67\%) y 4,45 (93,75\%). El menor valor promedio se logró en la escarificación física en el tratamiento 8 con 2,28(4,17\%).

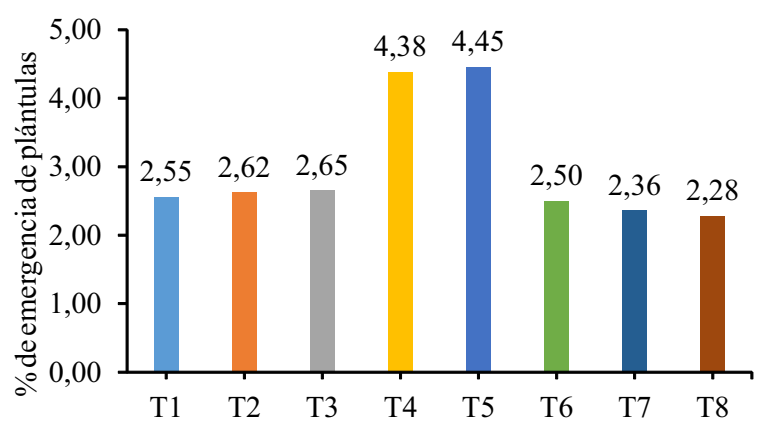

Figura 1. Porcentaje de emergencia acumulada en plántulas de tara.

\section{Altura de plántulas}

Para la variable altura de plántulas en la Tabla 3, de acuerdo a la prueba estadística de Duncan, que el análisis de varianza ha determinado diferencias altamente significativas $(p<0,05)$ entre los tratamientos de escarificación con coeficiente de variación de 28,51\%. 
Tabla 3. Prueba de medias para la variable altura de plántulas.

\begin{tabular}{ccc}
\hline Tratamiento & Media & Rango \\
\hline T5 & 8,97 & $\mathrm{~A}$ \\
T4 & 7,85 & $\mathrm{~A}$ \\
T8 & 5,47 & $\mathrm{~B}$ \\
T3 & 5,40 & $\mathrm{~B}$ \\
T2 & 5,36 & $\mathrm{~B}$ \\
T1 & 4,70 & $\mathrm{~B}$ \\
T7 & 4,50 & $\mathrm{~B}$ \\
T6 & 4.30 & $\mathrm{~B}$ \\
\hline
\end{tabular}

En la Figura 2 se puede apreciar que los tratamientos 4 y 5 con escarificación mecánica presentaron los mayores promedios de altura de plántula con 7,85 y $8,97 \mathrm{~cm}$ superando ambos a los demás tratamientos con escarificación física.

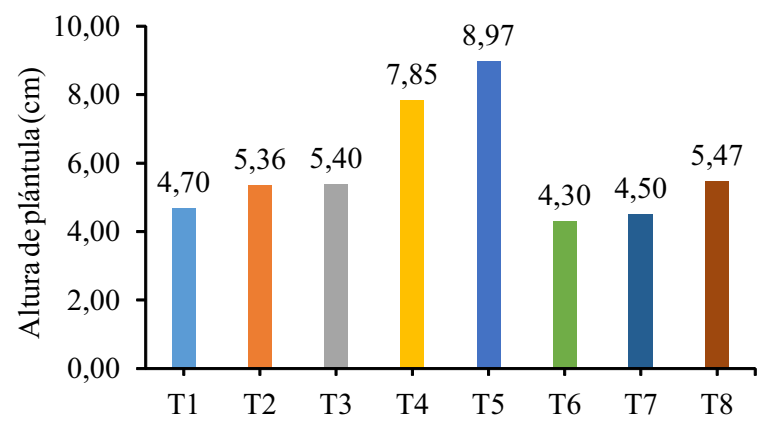

Figura 2. Altura de plántulas de tara.

\section{Número de hojas}

Para la variable número de hojas, de acuerdo al análisis de varianza existe diferencias altamente significativa $(\mathrm{p}<0,05)$ entre los tratamientos de escarificación con $27,5 \%$ de coeficiente de variación según el análisis estadístico de Duncan(Tabla4).

Tabla 4. Prueba de medias para la variable número de hojas.

\begin{tabular}{ccc}
\hline Tratamiento & Media & Rango \\
\hline T5 & 3,87 & $\mathrm{~A}$ \\
T4 & 3,70 & $\mathrm{~A}$ \\
T3 & 3,05 & $\mathrm{~B}$ \\
T2 & 2,77 & $\mathrm{~B}$ \\
T1 & 2,27 & $\mathrm{C}$ \\
T6 & 2,00 & $\mathrm{C}$ \\
T7 & 2,00 & $\mathrm{C}$ \\
T8 & 2,00 & $\mathrm{C}$ \\
\hline
\end{tabular}

En la Figura 3 se comprueba que los tratamiento 4 y 5 han presentado los mayores promedios de número de hojas con 3,70 y 3,87. En cambio el menor número de hojas correspondió a los tratamientos 1, 6, 7 y 8 .

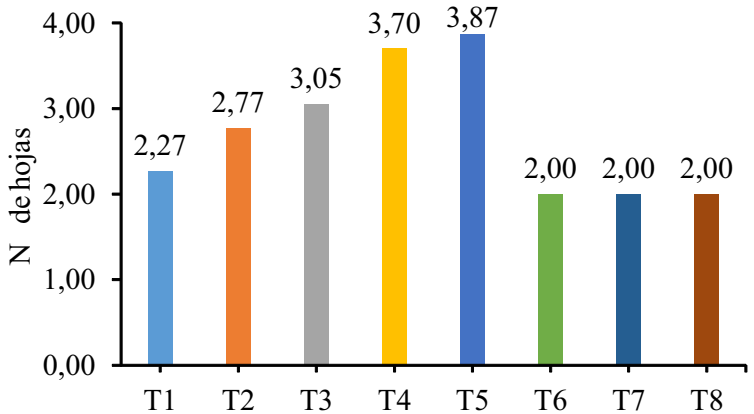

Figura 3. Número de hojas en plántulas de tara.

\section{Longitud de raíz}

De acuerdo al análisis de varianza mediante la prueba de Duncan señala que existen diferencias significativas $(\mathrm{p}<0,05)$ entre los tratamientos de escarificación con 23,16\% de coeficiente de variación (Tabla 5).

Tabla 5. Prueba de medias para la variable longitud de raíz.

\begin{tabular}{ccc}
\hline Tratamiento & Media & Rango \\
\hline T5 & 15,43 & $\mathrm{~A}$ \\
T4 & 15,05 & $\mathrm{~A}$ \\
T1 & 13,58 & $\mathrm{AB}$ \\
T3 & 12,33 & $\mathrm{AB}$ \\
T2 & 11,82 & $\mathrm{AB}$ \\
T8 & 11,11 & $\mathrm{BC}$ \\
T6 & 10,87 & $\mathrm{BC}$ \\
T7 & 8,05 & $\mathrm{C}$ \\
\hline
\end{tabular}

En la Figura 4 se puede observar que los tratamientos 4 y 5 con escarificación mecánica obtuvieron los promedios más altos de longitud de raíz con 15,05 y 15,43 $\mathrm{cm}$ respectivamente. Mientras tanto el menor promedio se obtuvo mediante la escarificación física en el tratamiento $7 \operatorname{con} 8,05 \mathrm{~cm}$.

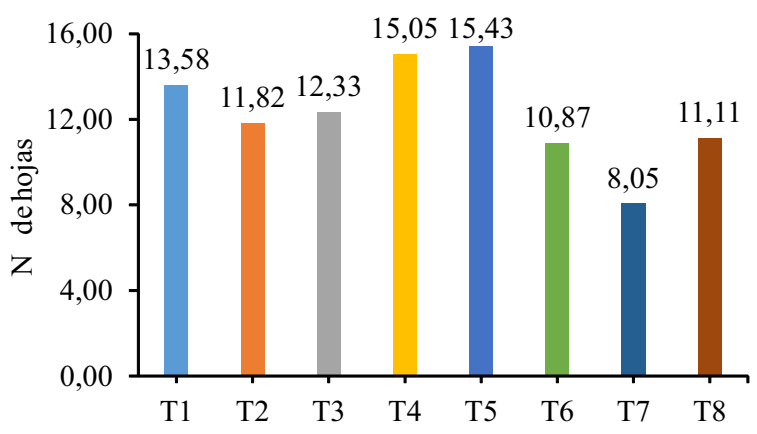

Figura 4. Longitud de raíz en plántulas de tara

\section{Índice de velocidad de emergencia}

Para la variable de índice de velocidad de emergencia de acuerdo al análisis de varianza mediante la prueba de Duncan $(\mathrm{p}<0,05)$ presentaron diferencias altamente 
significativas, igualmente para garantizar que los datos pertenezcan a una distribución normal fueron transformados a la función $\log (x+1)$ con coeficiente de variación de 27,29\% (Tabla 6).

Tabla 6. Prueba de medias para la variable el índice de velocidad de emergencia de altura de plántulas

\begin{tabular}{cccc}
\hline Tratamiento & Media & Media $\log (\mathbf{x}+\mathbf{1})$ & Rango \\
\hline T5 & 14,85 & 1,98 & $\mathrm{~A}$ \\
T4 & 14,16 & 1,97 & $\mathrm{~A}$ \\
T3 & 2,03 & 1,47 & $\mathrm{~B}$ \\
T2 & 1,86 & 1,39 & $\mathrm{BC}$ \\
T1 & 1,54 & 1,33 & $\mathrm{C}$ \\
T6 & 1,24 & 1,32 & $\mathrm{C}$ \\
T7 & 0,46 & 0,93 & $\mathrm{D}$ \\
T8 & 0,28 & 0,77 & $\mathrm{E}$ \\
\hline
\end{tabular}

En la Figura 5 se muestra el índice de velocidad de emergencia donde los tratamientos que mejor respondieron fueron el 4 y 5 con escarificación mecánica donde presentaron valores promedios de 1,97 $(14,16)$ y $1,98(14,85)$ respectivamente. El menor valor promedio se presentó en la escarificación física en el tratamiento 8 con $0,77(0,28)$.

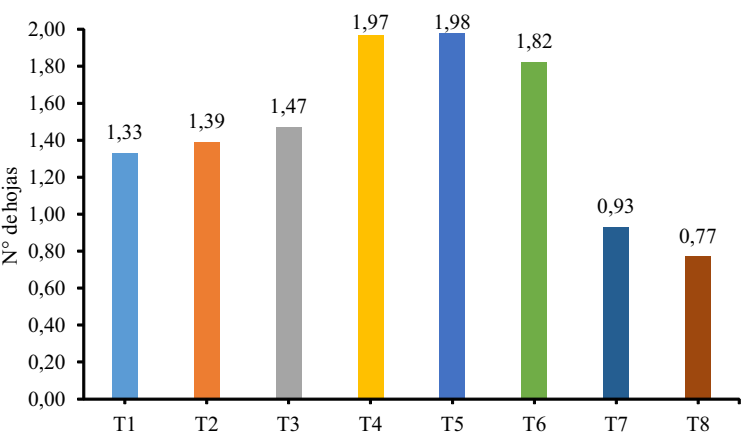

Figura 5. Índice de velocidad de emergencia acumulada en plántulas de tara.

\section{DISCUSIÓN}

Observando los resultados de la investigación, se pude determinar que la escarificación física tuvo mayor influencia sobre las características morfológicas y en el proceso de germinación en las plántulas de C. spinosa y además por haber eliminado la latencia en forma más rápida y práctica. Al aplicar el corte de la testa, se propició que la semilla absorba el agua del exterior de forma inmediata, y se active el proceso de germinación entre las 12 y 24 horas con valores del 93,75 \% y $91,67 \%$ de germinación. Estos resultados, concuerdan con los de otras investigaciones, donde la escarificación mecánica a través de material rugoso como la lija se obtuvo el 98,90 \% de germinación y/o la emergencia de plántulas en la especie Caesalpinia platyloba (Sánchez-Soto et al., 2016). Igualmente, en el estudio de Susanibar (2013), se aplicó una escarificación mecánica y física, primero picando las semillas de Caesalpinia espinosa y luego remojando las mismas en agua fría por un período de entre 12 y 24 horas, logrando alcanzar el 99,33\% y el $99 \%$ de germinación. Sin embargo, remojando las semillas en agua caliente, se daña el embrión, puesto que se dan los porcentajes de germinación, con promedios del 37,33 $\%$ y el 3,67\%, acercándose a los resultados obtenidos en nuestra investigación, alcanzando porcentajes de germinación del $20,42 \%$, el 9,58 \% y el 4,17\% en los tratamientos 6,7 y 8 .

Para las variables de altura de planta y de número de hojas el picado, el remojado de las semillas en agua fría influenció en la obtención de los mayores promedios con 6,48 cm y 8 hojas para ambos períodos de 12 y 24 horas. Resultados parecidos se observan en los tratamientos 4 y 5 con la escarificación mecánica con promedios superiores de $7,85 \mathrm{~cm}$ y $8,97 \mathrm{~cm}$ de altura de planta, 3,87 y 3,70 número de hojas. Igualmente en la longitud de raíz se alcanzó promedios superiores de $15,43 \mathrm{~cm}$ y $15,05 \mathrm{~cm}$, demostrando que las semillas cortadas y embebidas en agua de 12 a 24 horas influyeron en el crecimiento e incremento del área foliar de las plántulas de tara.

También se puede evidenciar el éxito de la escarificación mecánica en especies endémicas de bosques como C. glabrata donde la respuesta germinativa estuvo ligada directamente a los tratamientos alcanzando $96 \%$ de germinación acumulada mediante la ruptura de la testa. La alta respuesta germinativa, puede deberse a que la planta madre proporcionó la misma cantidad de recursos en la producción de semillas (Romero-Saritama et al., 2016).

La tara, por poseer una testa dura y una cutícula impermeable, pudo evidenciar diferencias significativas entre tratamientos en cuanto a la imbibición al aplicar la escarificación física (remojo en agua fría por 3,5 y 7 días). Parece ser, que el tiempo de remojo no 
fue suficiente para incrementar el proceso de germinación, por lo que se requirió un período mayor de remojado ya que la testa dura limitó la capacidad de absorción de agua que evidencia por ende la latencia física de las semillas (Baskin y Baskin, 2013)

Otro aspecto importante es el índice de velocidad de emergencia que se determinó a partir de la germinación de las plántulas, en este sentido el corte de la testa como tratamiento de escarificación mecánica fue un mecanismo que incrementó la aceleración en el proceso germinativo estableciéndose una relación más directa con los tratamientos 4 y 5 con valores de 14.16 y 14.85 quienes presentaron un mejor comportamiento. Esto se demuestra también en la escarificación física como uno de los mejores tratamientos donde semillas de Bauhinia tarapotensis "Patevaca" fueron sometidas a imbibición en agua a temperatura ambiente durante 24 horas logrando en este caso un promedio de 4.34 de valor en el índice de velocidad de germinación hasta los 19 días (Méndez-Vargas et al., 2015). Los resultados varían con respecto en ambos casos pudiendo ser por el tipo de escarificación, el tiempo de evaluación, la especie utilizada, el grosor y contenido de la testa; pero se debe enfatizar la relación directa que existe entre la velocidad de emergencia y/o germinativa con el tipo de tratamiento germinativo que se aplique a las semillas, dependiendo de esto se va obtener plántulas más vigorosas.

\section{CONCLUSIONES}

Los mejores resultados obtenidos en el porcentaje de emergencia de plántulas de C. spinosa fueron en los tratamientos 4 y 5 con escarificación mecánica alcanzando promedios de 91,67 \% y 93,75\%, ya que se eliminó la latencia física y el embrión tuvo mayor facilidad para emerger, siendo un método práctico que se puede utilizar para la propagación de esta especie. En las variables morfológicas de altura de plántula, número de hojas y longitud de raíz fueron influenciados notoriamente por los tratamientos de escarificación mecánica, demostrándose así que las semillas sin alteraciones internas en su estructura y composición tendrán una mejor respuesta durante la germinación y emergencia de las plántulas.

Los tratamientos de escarificación física no influyeron adecuadamente en las características morfológicas y germinativas debido al daño que sufrieron las semillas al ser sumergidas en agua hervida y al menor tiempo de remojo, para lo cual se debería sugerir sumergir las semillas en tiempos menores a 1 minuto y remojarlas por un período mayor a una semana.

El índice de velocidad de emergencia viene a ser un indicador del vigor de las semillas lo que determinó de acuerdo a los resultados la relación de los tratamientos de escarificación en el proceso germinativo y de emergencia en las plántulas de tara.

\section{REFERENCIAS BIBLIOGRÁFICAS}

Aguilar-Galvez, A., G. Noratto, F. Chambi, F. Debaste, y D. Campos. 2014. "Potential of Tara (Caesalpinia spinosa) Gallotannins and Hydrolysates as Natural Antibacterial Compounds." Food Chemistry 156: 301-4. doi:10.1016/j.foodchem.2014.01.110.

Alvarez-Aquino, C., L. Barradas-Sánchez, O. PonceGonzález y G. Williams-Linera. 2014. "Soil Seed Bank, Seed Removal y Germination in a Seasonally Dry Tropical Forest in Veracruz, Mexico.” Botanical Sciences 92 (1): 111-21.

Atencio, L., R. Colmenares, M. Ramírez y D. Marcano. 2003. "Tratamientos Pregerminativos En Acacia San Francisco (Peltophorum pterocarpum) Fabaceae." Revista de La Facultad de Agronomía 20: 63-71. Recuperado de: http://www.scielo.org.ve/scielo.php?script $=\mathrm{s}$ c i_a r t t e x t \& p i d = S 0378 78182003000100007.

Baskin, C. C. y J. M. Baskin. 2013. Seeds Ecology, Biogeography y Evolution of Dormancy and Germination. Londres (Reino Unido): Acad e m i c P r e s s E $1 \mathrm{~s}$ e r vie r. doi:10.1017/CBO9781107415324.004.

Baskin, J. M. y C. C. Baskin. 2004. “A Classification System for Seed Dormancy." Seed Science 
Research 14: 1-16. doi:10.1079/ SSR2003150.

Bewley, J. D. 1997. "Seed Germination and Dormancy." The Plant Cell 9 (7): 1055-1066. doi:10.1105/tpc.9.7.1055.

Cárdenas, J. F. 2011. Morfología y Tratamientos Pregerminativos de Semillas de Granadilla (Passiflora ligularis Juss). Tesis de Maestría. Universidad Nacional de Colombia. Bogotá (Colombia).

Castell-Escuer, J. C. 2012. Tara (Coesalpinia spinosa): The Sustainable Source of Tannins for Innovative Tanning Processes. Tesis de Doctorado. Universitat Politécnica de Catalunya. Barcelona (España).

Cordero, I., B. Ruiz-Díez, L. Balaguer, A. Richter, J. J. Pueyo, y A. Rincón. 2017. "Rhizospheric Microbial Community of Caesalpinia spinosa (Mol.) Kuntze in Conserved and Deforested Zones of the Atiquipa Fog Forest in Peru." Applied Soil Ecology 114: 132-41. doi:10.1016/j.apsoil.2017.02.015.

Coronel de Renolfi, M., G. Cardona, M. Ewens, y E. Ibarra. 2013. "Productividad y Requerimientos de Mano de Obra En La Producción Comercial de Plantines de Algarrobo Blanco (Prosopis alba). El Caso Del Vivero Forestal de La Estación Experimental Fernández, Santiago Del Estero Productivity." Quebracho 21 (1-2): 26-38.

Dodds, R. M. 2015. Evaluación de Proyecto de Pre Factibilidad Para La Plantación e Instalación de Una Planta Piloto de Extracción de Harina y Goma de Tara ( Caesalpinia spinosa ) En Chile. Tesis de Ingeniería. Universidad de Chile. Santiago de Chile (Chile).

Doria, J. 2010. “Generalidades Sobre Las Semillas: Su Producción, Conservación y Almacenamiento." Cultivos Tropicales 31 (1): 74-85. doi:10.1016/S0168-6496(98)00035-X.

Dostert, N., J. Roque, G. Brokamp, A. Cano, M. Weigend, y M. I La Torre. 2009. Factsheet : Datos
Botánicos de Tara. Lima (Perú): Universidad Nacional Mayor de San Marcos.

Finch-Savage, W. E., y G. Leubner-Metzger. 2006. "Seed Dormancy and the Control of Germination.” The New Phytologist 171 (3): 501-23. doi:10.1111/j.1469-8137.2006.01787.x.

Méndez-Vargas, D. F., L. D. Trujillo-Vela, N. CabreraLópez, y C. Becerra-Ordoñez. 2015. "Evaluación de Métodos de Escarificación En Germinación, Crecimiento y Producción Biomasa de Bauhinia Tarapotensis Benth Diego.” Momentos de Ciencia 12 (1): 54-59.

Oliva, M., F. Vacalla, D. Pérez, y A. Tucto. 2014. Vivero Forestal Para Producción de Plantones de Especies Forestales Nativas: Experiencia En Molinopampa, Amazonas - Perú. Chachapoyas (Perú): ITTO/MINAGRI/SERFOR/IIAP.

Ortega-Baes, P., M. L. De Viana, G. Larenas, y M. Saravia. 2001. "Germinación de Semillas de Caesalpinia paraguariensis (Fabaceae): Agentes Escarificadores y Efecto Del Ganado." Revista de Biología Tropical 49 (1): $1-15$.

Patel, J. K., N. M. Patel, y R. L. Shiyani. 2001. “Coefficient of Variation in Field Experiments and Yardstick Thereof - An Empirical Study." Current Science 81 (9): 1163-64.

Romero-Saritama, J. M., V. B. Orellana-Armijos, y M. J. Balseca-Ruiz. 2016. "Morphology, Imbibition and Germination of Caesalpinia glabrata Kunth (Fabaceae) Seeds Distributed in a Tropical Dry Forest." Revista Chapingo Serie Zonas Áridas 15 (2): 89-101. doi:10.5154/r.rchsza.2016.05.004.

Rossini-Oliva, S. R., B. Valdés, M. C. Andrés, F. Márquez-Campón, y M. Bueso-López. 2006. "Especies Americanas De Fabaceae Y Bignoniaceae Cultivadas En Sevilla ( So España ).” Lagascalia 129: 119-29.

Sánchez-Paz, Y., y M. Ramírez-Villalobos. 2006. “Tratamientos Pregerminativos En Semillas de Leucaena leucocephala (Lam.) de Wit. y 
Prosopis juliflora (Sw.) DC." Revista de La Facultad de Agronomía 23 (3): 114-24. Recu$\mathrm{p}$ e $\mathrm{r}$ a d o d e

http://200.74.222.178/index.php/agronomia/ article/view/11392\%0Ahttp:/www.scielo. org.ve/scielo.php?script=sci_art text\&pid=S0378-78182006000100010.

Sánchez-Soto, B. H., E. Pacheco-Aispuro, Á. ReyesOlivas, G. A. Lugo-García, P. CasillasÁlvarez, y C. P. Sauceda-Acosta. 2016. "Ruptura de Latencia Física En Semillas de Caesalpinia platyloba $\mathrm{S}$. Watson.” INTERCIENCIA 41 (10): 691-95.

Susanibar, T. D. 2013. Evaluación de Emergencia de Semillas Escarificadas de Caesalpinea Spinosa 'Tara' Por Métodos Mecánicos y Fisicos. Tesis de Grado. Universidad Nacional José Faustino Sanchez Carrion. Huacho (Peru).

Trigiano, R. N., y D. J. Gray. 2011. Plant Tissue Culture, Development y Biotechnology. Boca Raton ( E E U U) C R C Pres s. doi:10.1017/CBO9781107415324.004. 